VOL 2 (2018) NO 4 - 2

e-ISSN : 2549-9904

ISSN : 2549-9610

INTERNATIONAL JOURNAL ON INFORMATICS VISUALIZATION

\title{
Network Security Assessment Using Internal Network Penetration Testing Methodology
}

\author{
Deni Satria ${ }^{\#}$, Alde Alanda ${ }^{\#}$, Aldo Erianda ${ }^{\#}$, Deddy Prayama ${ }^{\#}$ \\ \#Information Technology Department, Politeknik Negeri Padang, Indonesia \\ E-mail: deni@pnp.ac.id
}

\begin{abstract}
The development of information technology is a new challenge for computer network security systems and the information contained in it, the level of awareness of the importance of network security systems is still very low. according to a survey conducted by Symantec, the desire to renew an existing security system within a year within a company has the result that only $13 \%$ of respondents consider changes to the security system to be important from a total of 3,300 companies worldwide as respondents. This lack of awareness results in the emergence of security holes that can be used by crackers to enter and disrupt the stability of the system. Every year cyber-attacks increase significantly, so that every year there is a need to improve the security of the existing system. Based on that, a method is needed to periodically assess system and network security by using penetrarion testing methods to obtain any vulnerabilities that exist on the network and on a system so as to increase security and minimize theft or loss of important data. Testing is carried out by using internal network penetration testing method which tests using 5 types of attacks. From the results of the tests, each system has a security risk of $\mathbf{2 0 - 8 0 \%}$. From the results of these tests it can be concluded that each system has a security vulnerability that can be attacked.
\end{abstract}

Keywords - Penetration testing, network security, vulnerability.

\section{INTRODUCTION}

The development of information technology has an important role in people's lives. With the development of technology that is always undergoing change, making information security an important factor (Mason, 1986). Once the importance of the value of information often causes the information to be accessed only by certain people who have authority. So that the fall of information into the hands of unauthorized parties can cause harm to the information owner. For example, a lot of important information in a company is only allowed to be known by certain people in the company, such as information about products that are under development, algorithms and techniques used to produce these products. For this reason, the security of the information system used must be guaranteed and in accordance with existing standards.

The development of information technology is a new challenge for computer network security and information systems, according to a survey conducted by Symantec, the level of awareness of the desire to renew an existing security system within a year within a company gets results that only $13 \%$ of respondents consider change the security system is important from a total of 3,300 companies worldwide as respondents (Symantec State of Security Survey, 2011). This lack of awareness results in the emergence of security holes that can be used by crackers to enter and disrupt the stability of the system.

Cyber attacks have caused various personal data thefts. In government offices there have been nearly 21.5 million people who have experienced theft of data from office computers. In addition to government offices, attacks also occur in banks in the world. Cyber attacks have started since the end of 2013 and have stolen about 1 trillion US Dollars. More than 100 banks in the world from 30 countries affected by cyber attacks. The hacker installs spyware into a computer that is used by bank employees and observes the workings of bank employees and secretly transfers to bank accounts that are used for theft of money. According to brearchieveindex.com from 2013 to June 2015 there have been more than 3 billion lost and stolen data involving all types of people such as retail, government, education, financial and others.

\section{LITERATURE REVIEW}

\section{A. Network Security}

Network security is very important to monitor network access and prevent unauthorized use of network resources. Network security tasks are controlled by the network 
administrator. Security aspects defined by five points, namely Confidentiality, require that information can only be accessed by those who have authority. Integrity, requires that information can only be changed by those who have the authority, availability, requires that information be available to parties who have the authority when needed, authentication, requires that the sender of an information be identified correctly and there is a guarantee that the identity obtained is not false. Nonrepudiation, requires that both senders and recipients of information cannot deny sending and receiving messages.

\section{B. Penetration Testing}

Penetration Testing is a method used to evaluate the security of a system or computer network by performing an attack simulation. In the OWASP methodology Web Application Security Testing focuses only on the security of web applications, where the process involves actively analyzing web applications, to find weaknesses, technical defects, and weaknesses. Security issues that have been discovered will be given to the system owner, which is included with a report that contains information about the estimated impacts that arise as well as technical solutions to these problems.

Penetration testing has proven effective in helping to deal with security issues on the network. Penetration testing techniques are not only aimed at applications, but can also be applied to networks, and operating systems, where the main purpose is to find and then try to exploit vulnerabilities that are known or detected in previous evaluations contained in certain technologies

There are 3 types of penetration testing, namely:

1. Black-Box

Penetration testing without knowing what systems or networks are used by an organization

2. White-Box

Penetration testing by knowing the infrastructure information that is in an organization

3. Grey-Box

It is a penetration test that combines blax-box with white box

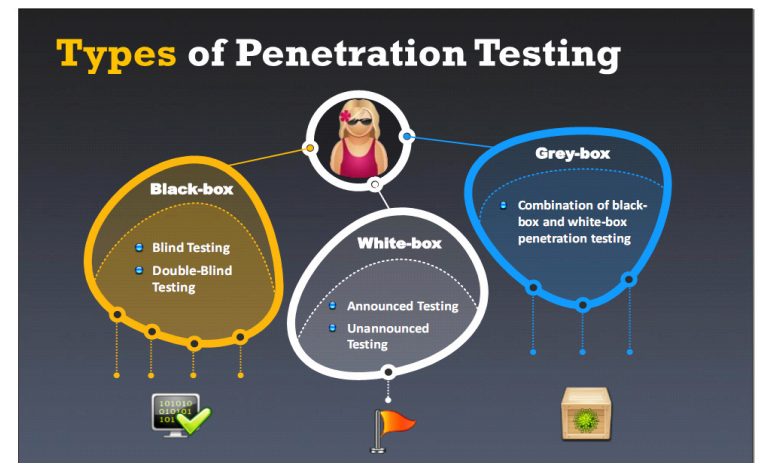

Fig. 1. Types of Penetration Testing

\section{METHODOLOGY}

This research uses internal network penetration testing method. The steps that will be carried out in this research can be seen in the picture below

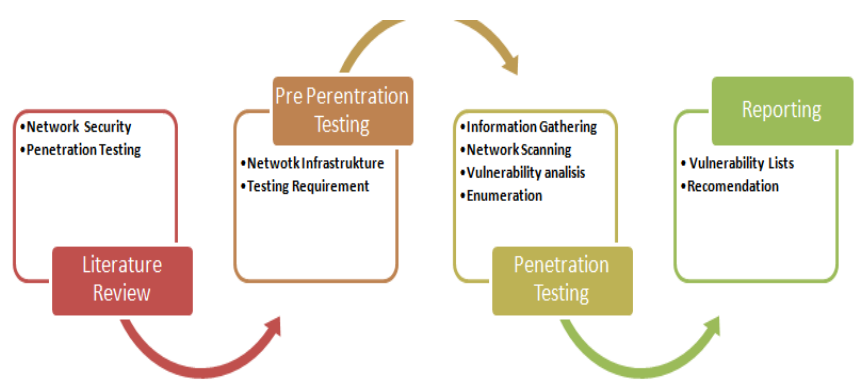

Fig. 2 Methodology

a. Literature Review by reading and understanding the literature in the form of books, sites, and scientific works related to network security and penetration testing

b. In the pre penetration testing stage, network security analysis is performed and then what components are needed to be assessed in the network.

c. Penetration Testing, at this stage assessment is carried out by using various tests on security loopholes contained in the system and exploiting the current system.

d. Reporting, after completing the assessment of the security of the system, then make a report based on the results of the assessment and provide recommendations on the security risks found in the system.

\section{PENETRATION TESTING}

\section{a. Scope of Project}

Scope of testing on servers on the internal network there are 5 servers in one network. IP network of internal network is X.X.X.0/24. The following is the list of IP servers that will be tested.

TABLE I

LIST OF IP SERVER

\begin{tabular}{|l|c|}
\hline \multicolumn{2}{|c|}{ IP ADDRESS } \\
\hline S1 & X.X.X.68 \\
\hline S2 & X.X.X.77 \\
\hline S3 & X.X.X.103 \\
\hline S4 & X.X.X.123 \\
\hline S5 & X.X.X.152 \\
\hline
\end{tabular}

The above IP address is taken based on scanning the host through the internal network using the white box method. Each of these IP represents each existing server. only 5 machines are taken as examples of testing.

Some of the things tested in the server penetration testing on the internal network are as follows.

TABLE III

VULNERABLE INFORMATION

\begin{tabular}{|c|c|}
\hline ID Vulnerable & Information \\
\hline V1 & Vulnerable web application \\
\hline V2 & Vulnerable of Plugin \\
\hline V3 & Default Username and Password \\
\hline V4 & DOS \\
\hline V5 & Cross Site ScrIPting \\
\hline
\end{tabular}


The ID is used to provide an identity for the security holes that are found later in the system. Its important applied to evaluate system or server based on internal network.

b. Information Gathering

This information is obtained using Zenmap tools with banner grabbing methods to get all information about the machines.

TABLE IIIII

INFORMATION S1 MACHINE

\begin{tabular}{|l|l|}
\hline IP Address & X.X.X.68 \\
\hline Operating System & Linux 3.10 \\
\hline Device Type & Web Server \\
\hline Hostname & XXX \\
\hline Active Port & Services \\
\hline 21 & FTP (ProFTPD or KnfTPD) \\
\hline 22,2222 & SSH (OpenSSH 7.4 (protocol 2.0)) \\
\hline 25,587 & SMTP (Postfix smtpd) \\
\hline 80,10000 & HTTP (Apache httpd 2.4.6 (PHP 5.4.16)) \\
\hline 110,995 & Pop3 (Dovecot pop3d) \\
\hline 143,993 & IMAP (Dovecot imapd) \\
\hline 443 & HTTPS (Apache/2.4.6) \\
\hline 53 & Domain (ISC BIND 9.9.4) \\
\hline
\end{tabular}

TABLE IVV

INFORMATION S2 MACHINE

\begin{tabular}{|l|l|}
\hline IP Address & X.X.X.77 \\
\hline $\begin{array}{l}\text { Operating } \\
\text { System }\end{array}$ & Linux 3.10 \\
\hline Hostname & Sistem Informasi Presensi \\
\hline Device Type & Server \\
\hline Active Port & Services \\
\hline 222 & OpenSSH 6.6.1p1 Ubuntu 2ubuntu2 \\
\hline 53 & ISC BIND 9.9.5-3 (Ubuntu Linux) \\
\hline 80 & Apache httpd 2.4.7 ((Ubuntu)) \\
\hline 3306 & MySQL 5.5.38-0ubuntu0.14.04.1 \\
\hline
\end{tabular}

TABLE V

INFORMATION S3 MACHINE

\begin{tabular}{|l|l|}
\hline IP Address & X.X.X.103 \\
\hline Operating System & 3com Embedded \\
\hline Device Type & Switch \\
\hline Hostname & Baseline Switch \\
\hline Active Port & Services \\
\hline 80 & $\begin{array}{l}\text { GoAhead WebServer (LinkSys } \\
\text { SLM2024 or SRW2008 - } \\
\text { SRW2016 switch http config) }\end{array}$ \\
\hline 443 & $\begin{array}{l}\text { ss1/http GoAhead WebServer } \\
\text { (Linksys SRW2024 switch http } \\
\text { config) }\end{array}$ \\
\hline
\end{tabular}

TABLE VI

INFORMATION S4 MACHINE

\begin{tabular}{|l|l|}
\hline IP Address & X.X.X.123 \\
\hline Operating System & Hp Laser Jet \\
\hline Device Type & Printer \\
\hline Hostname & HP LaserJet M3027 MFP Series \\
\hline Active Port & Services \\
\hline 21 & HP FTP Print Server 3.0 \\
\hline 23 & HP Jetdirect Telnet \\
\hline $80,280,631$ & HP-ChaiSOE 1.0 \\
\hline
\end{tabular}

TABLE VII

INFORMATION S5 MACHINE

\begin{tabular}{|l|l|}
\hline IP Address & X.X.X.152 \\
\hline Operating System & Windows Xp (x32) \\
\hline Device Type & Host \\
\hline Hostname & Splash.php \\
\hline Active Port & Services \\
\hline 80 & $\begin{array}{l}\text { Apache httpd 2.4.3 ((Win32) } \\
\text { OpenSSL/1.0.1c PHP/5.4.7) }\end{array}$ \\
\hline 443 & $\begin{array}{l}\text { Apache httpd 2.4.3 ((Win32) } \\
\text { OpenSSL/1.0.1c PHP/5.4.7) }\end{array}$ \\
\hline \multirow{2}{*}{49152} & $\begin{array}{l}\text { Portable SDK for UPnP devices } \\
1.4 .7(\text { Windows 6.2.9200 2; } \\
\text { UPnP 1.0) }\end{array}$ \\
\hline
\end{tabular}

\section{c. Penetration Tesing}

1. Penetration Testing on S1 Machine

After getting information about the target, proceed with the vulnerability analysis method, which analyzes the the weaknesses of the system that can be exploited during the attack session. based on the information above, web applications use WordPress with version 4.24. then the examiner will enter the WordPress Login Page for enumeration to get information about the username and password.

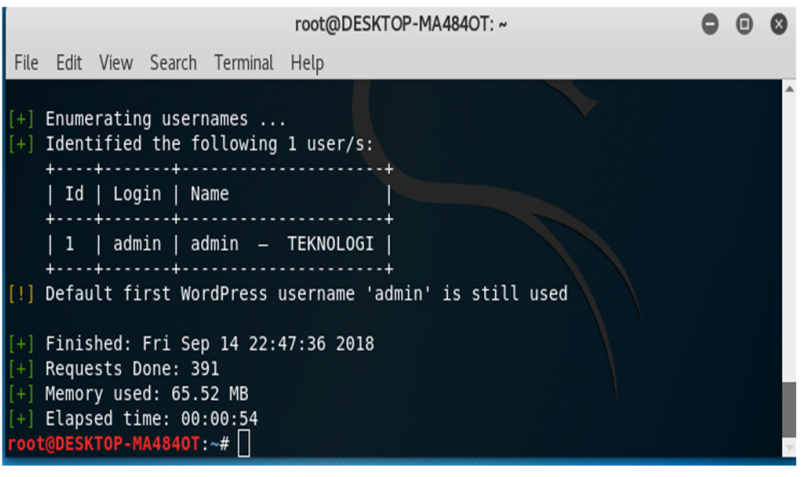

Fig. 3 Enumeration Username Using Wpscan

In this case, the username is obtained, but the password cannot be indicated because the combination of characters in the password is difficult to hack. The test continued by finding the weaknesses of the web server using the Owasp Zap tool. 


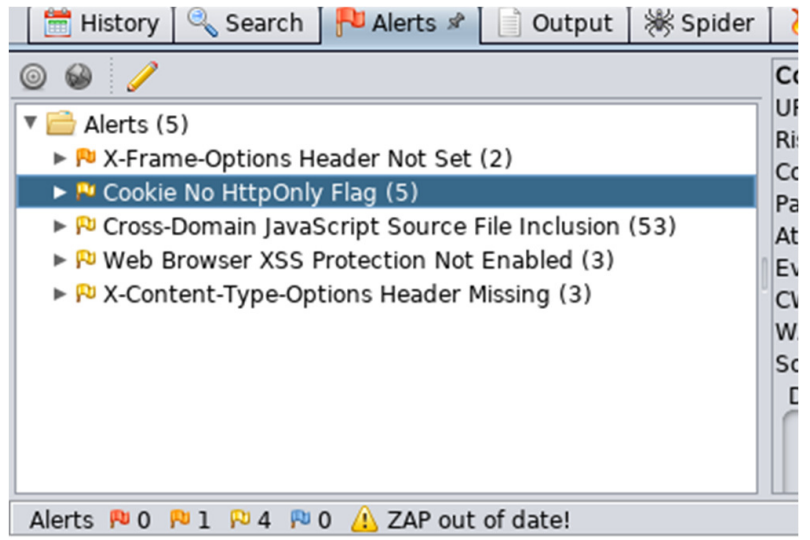

Fig. 4 Finding Vulnerable Using OWASP Zap Tools

Based on the picture above, found 5 things that become a weakness in the web scripting section that can be exploited later using the Cross Site Scripting attack type.

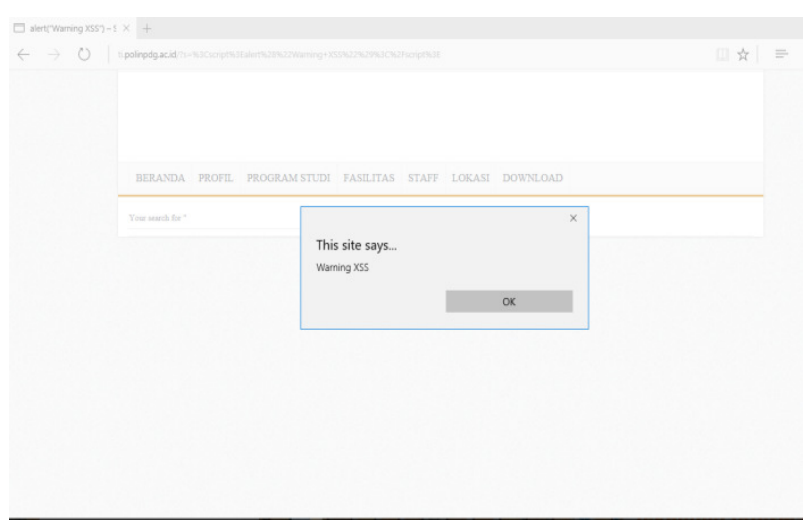

Fig. 5 XSS Attack on TI Web Server

The next test is DOS attack. Based on information that the machine use XMLRPC Protocol in the application, the testers can attack through the protocol to overload and flood the server by sending a lot of requests in a small time. The attack was carried out using metasploit tools with wordpress xmlrpc dos module and make lot request to server.

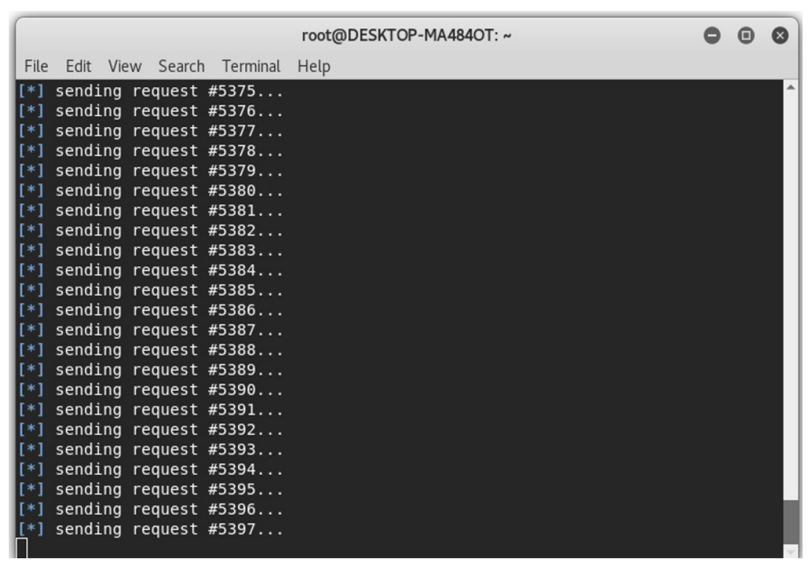

Fig. 6 DOS Attacking using Metasploit Tools

After the attack was carried out a lot of network traffic was drawn on the following etherape tools.

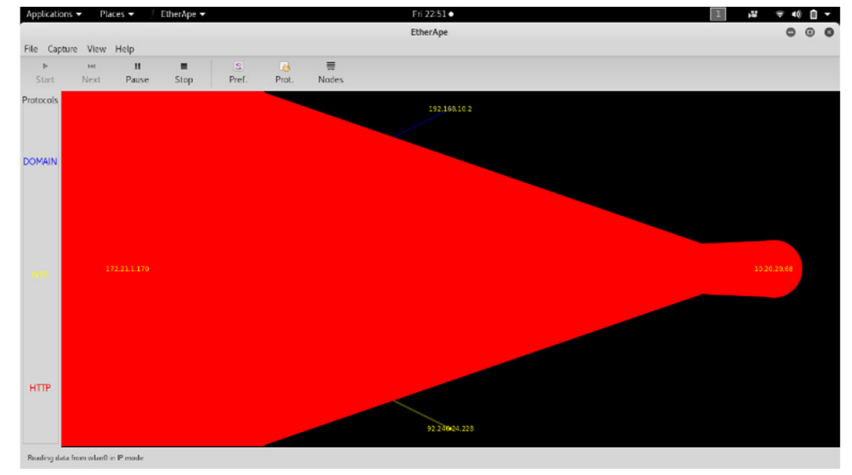

Fig. 7 Network Traffic Monitoring Using Etherape Tools

After perform DOS attack, the database connection on server becomes down. consequently the web cannot be accessed for a while.

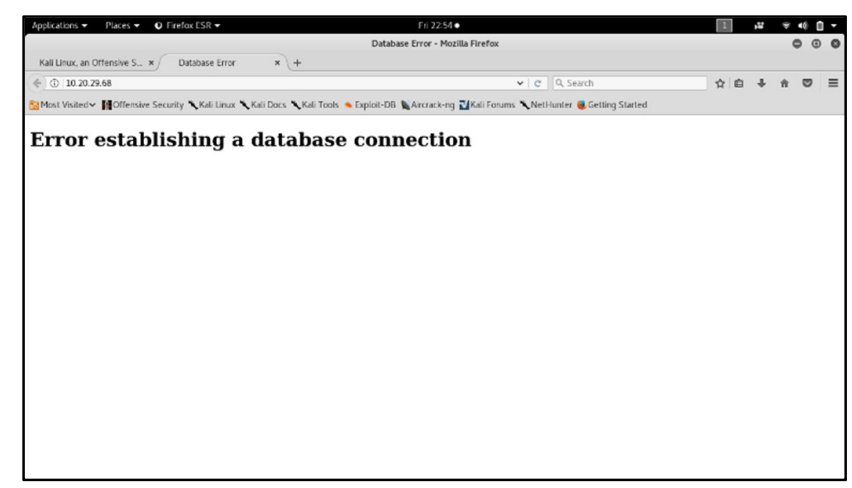

Fig. 8 Error Database Connection

TABLE VIII

RECOMMENDATION S1 MACHINE

\begin{tabular}{|c|c|}
\hline $\begin{array}{c}\text { ID of } \\
\text { Vulnerable } \\
\text { Detect }\end{array}$ & Recommendation \\
\hline V1 & $\begin{array}{l}\text { - Wordpress updates with the latest version to } \\
\text { minimize the discovery of security holes } \\
\text { The xmlrpc protocol should be hidden from } \\
\text { information obtained using wpscan tools } \\
\text { There are } 2 \text { ways to detect the occurrence of } \\
\text { XML-RPC attacks on wordpress, namely the } \\
\text { appearance of the message "error Connecting } \\
\text { to Database" when the website is accessed, } \\
\text { which indicates that the database is dead. In } \\
\text { addition there are a lot of "POST / xmlrpc.php } \\
\text { HTTP / 1.0" entries on the web server log }\end{array}$ \\
\hline $\mathrm{V} 2$ & $\begin{array}{l}\text { change the default username in the wordpres login } \\
\text { page }\end{array}$ \\
\hline V5 & $\begin{array}{l}\text { blocking of the IP from the sender who } \\
\text { sends a large number of requests at the same } \\
\text { time } \\
\text { Block access to the xmlrpc.php file. In this } \\
\text { way the XML-RPC attack will fail before } \\
\text { reaching its target of wordpress. The trick is } \\
\text { to edit the .htaccess file and add the } \\
\text { following lines: } \\
<\text { Files xmlrpc.php> } \\
\text { Order Deny, Allow } \\
\text { Deny from all } \\
\text { Allow from } 192.168 .1 .10 / 24 \\
\text { Satisfy all }\end{array}$ \\
\hline
\end{tabular}




\begin{tabular}{|l|l|}
\hline & $\begin{array}{l}\text { ErrorDocument } 403 \text { http://127.0.0.1/ } \\
</ \text { Files }> \\
\text { Allow IP 192.168.1.10/24 to approve the IP } \\
\text { administrator which is exemplified in the IP } \\
\text { above to configure }\end{array}$ \\
\hline V6 $\quad \begin{array}{l}\text { Hazard characters must be filtered from the } \\
\text { web application input } \\
\text { - }\end{array}$ & $\begin{array}{l}\text { Filters must be applied to ASCII and HEX } \\
\text { values } \\
\text { Update IDS (Intrusion Detection System) } \\
\text { which functions as the first signal provider if } \\
\text { an intruder tries to break into a computer } \\
\text { security system }\end{array}$ \\
\hline
\end{tabular}

\section{Penetration Testing on S2 Machine}

There are several weaknesses of security that can be exploited. The testing scenario on this server is to get data from the web application. These data can be used by person who are not responsible for the application. This can trigger integrity weaknesses and confidentiality data. Testers try to sign in using default authentication for the login page.

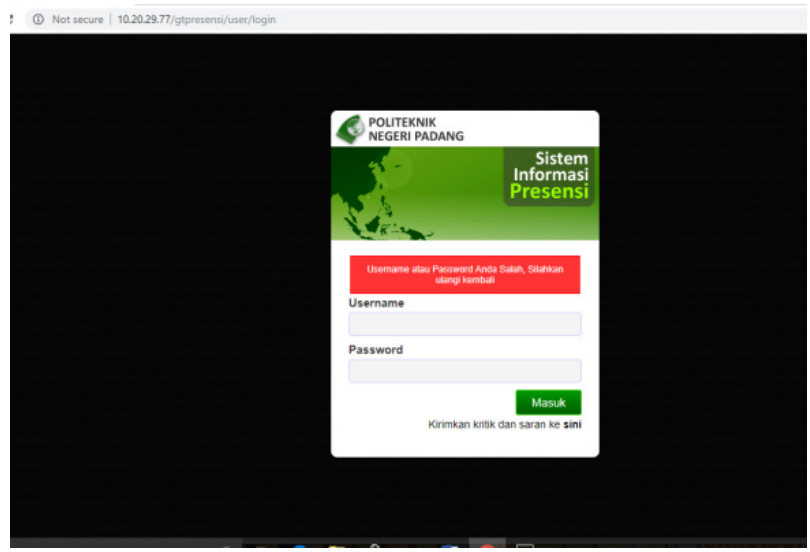

Fig. 9 Wrong Default Username and Password

The server contains weaknesses in XSS, the testers try to insert some characters to disguise authentication, so the login process can be forwarded in.

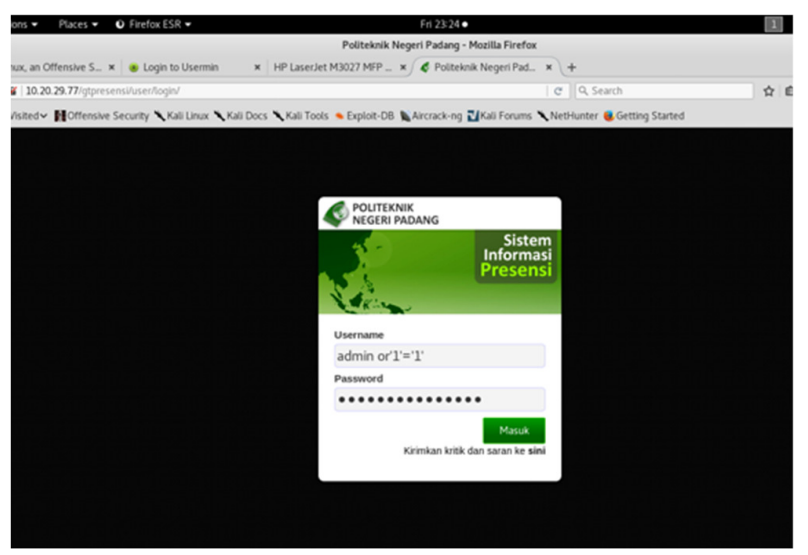

Fig.10 Inject Query to Login Page

Then the tester will try to retrieve data from the application based on the URL directory of the unprotected server. Access to the directory will be shown by the following picture.

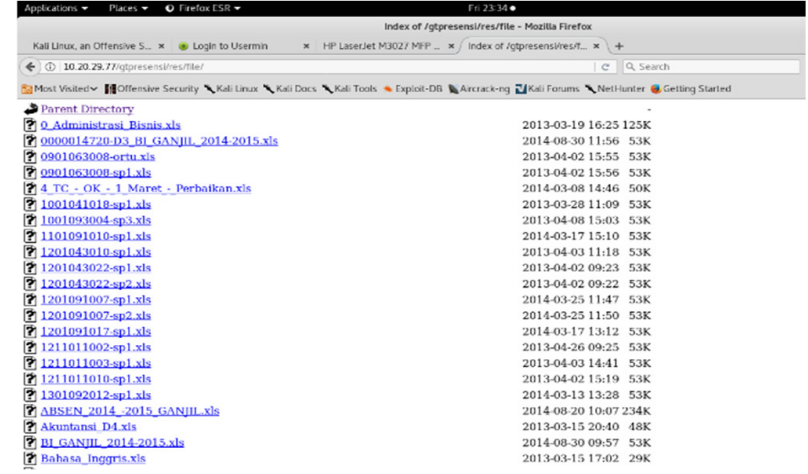

Fig. 11 Data from Directory Server

TABLE IX

EVALUATION AND RECOMMENDATION ON S2 MACHINE

\begin{tabular}{|c|l|}
\hline $\begin{array}{c}\text { ID of } \\
\text { Vulnerable } \\
\text { Detect }\end{array}$ & \multicolumn{1}{c|}{ Recommendation } \\
\hline V1 & $\begin{array}{l}\text { URL directory to access confidential data that is } \\
\text { not protected. This causes data exploitation. } \\
\text { therefore give authentication to access the URL } \\
\text { directory in the system }\end{array}$ \\
\hline V6 & $\begin{array}{l}\text { The server it can be injected using the XSS method } \\
\text { to disguise the username and password so that it } \\
\text { can escape session authentication }\end{array}$ \\
\hline
\end{tabular}

\section{Penetration Testing on S3 Machine}

For the S3 machine perform privilege escalation to login page using default username and password to entering the system.

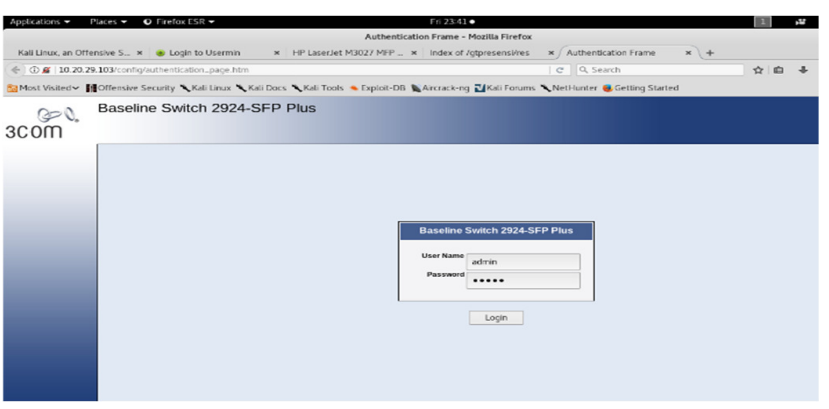

Figure 12. Testing Default Password on Baseline Switch Server

The tester successfully entered the system. This is a security gap that needs to be fixed, because if unauthorized user can enter the system, the settings can be damaged. Consequently the infrastructure and services on the network do not run optimally.

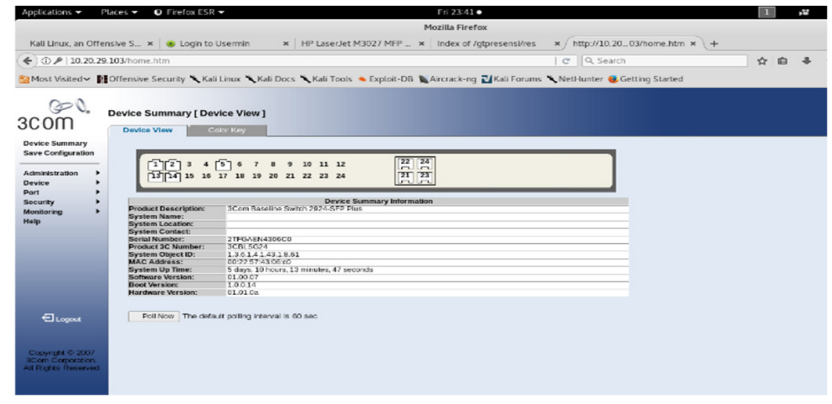

Fig.13 Successfully entered the system 


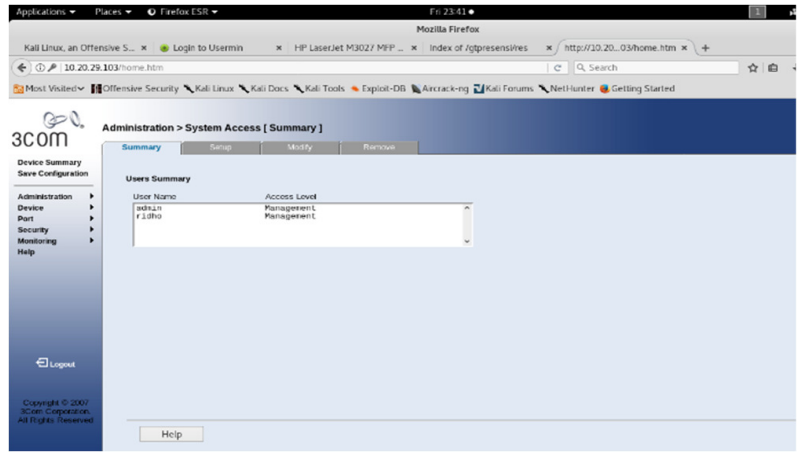

Fig. 14 Create New User in System

Testers can add users as administration to configure the system.

TABLE X

EVALUATION AND RECOMMENDATION FOR S3 MACHINE

\begin{tabular}{|c|l|}
\hline $\begin{array}{c}\text { ID of } \\
\text { Vulnerable } \\
\text { Detect }\end{array}$ & \multicolumn{1}{c|}{ Recommendation } \\
\hline V3 & $\begin{array}{l}\text { Change the username with a combination of } \\
\text { unusual characters making it difficult to numerate }\end{array}$ \\
\hline V4 & $\begin{array}{l}\text { Change the password with a combination of } \\
\text { unusual characters making it difficult to crack and } \\
\text { using encryption method to secure authentication }\end{array}$ \\
\hline
\end{tabular}

\section{Penetration Testing on S4 Machine}

After the examiner visits the IP through the browser, the system page does not have protection. So that can be accessed by anyone without protection. This is very dangerous for the system, because unauthorized user can configure and take over the system.
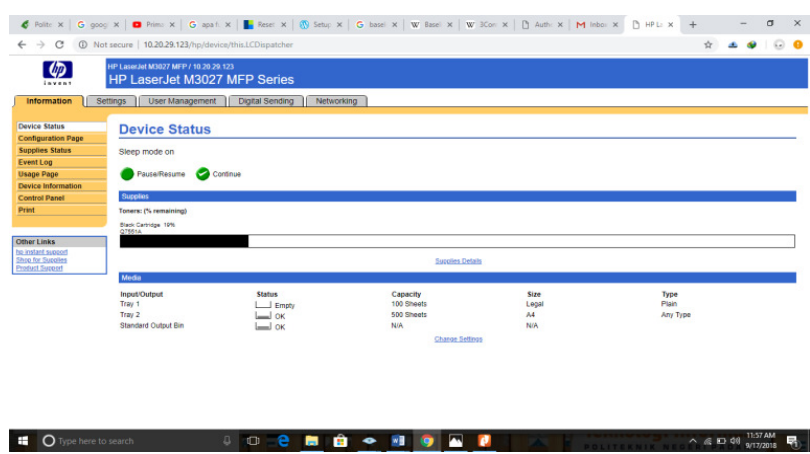

Fig. 15 Enter a System without protection

TABLE XI

EVALUATION AND RECOMMENDATION FOR S4 MACHINE

\begin{tabular}{|c|c|}
\hline $\begin{array}{c}\text { ID of } \\
\text { Vulnerable } \\
\text { Detect }\end{array}$ & \multicolumn{1}{c|}{ Recommendation } \\
\hline V1 & $\begin{array}{l}\text { There is no protection from the application } \\
\text { so that it can be accessed by anyone. } \\
\text { therefore add protection to the system such }\end{array}$ \\
\hline
\end{tabular}

\begin{tabular}{|l|l|}
\hline & $\begin{array}{l}\text { as authentication with a combination of } \\
\text { characters that are difficult to get by hackers }\end{array}$ \\
\hline
\end{tabular}

From the results of testing the level of security on the network, each device has a vulnerability level of $20 \%-80 \%$. $60 \%$ of devices that have been tested can be attacked using XSS attacks and $60 \%$ use the default username and password for the login process to the system

TABLE XII

SUMMARY OF FINDING

\begin{tabular}{|c|c|c|c|c|c|}
\hline $\begin{array}{c}\text { Mach } \\
\text { ines }\end{array}$ & DOS & $\begin{array}{c}\text { Cross } \\
\text { Site } \\
\text { Scriptin } \\
\text { g (XSS) }\end{array}$ & $\begin{array}{c}\text { Metasploi } \\
\mathrm{t}\end{array}$ & $\begin{array}{c}\text { Plugin } \\
\text { Attack }\end{array}$ & $\begin{array}{c}\text { Usernam } \\
\text { e and } \\
\text { Passwor } \\
\mathrm{d}\end{array}$ \\
\hline S1 & $\checkmark$ & $\checkmark$ & $\checkmark$ & $\checkmark$ & \\
\hline S2 & & $\checkmark$ & & & \\
\hline S3 & & $\checkmark$ & & & $\checkmark$ \\
\hline S4 & & & & & $\checkmark$ \\
\hline S5 & & & & & $\checkmark$ \\
\hline
\end{tabular}

\section{CONCLUSIONS}

From the results of penetration testing it can be concluded that almost every device has a weakness and can be attacked.. For this reason several recommendations are given so that device security can be improved.

\section{REFERENCES}

[1] Gupta, A., Kavita, \& Kirandeep, K. (2013). Vulnerability assessment and penetration testing. International Journal of Engineering Trends and Technology, 4(3), 328.

[2] Felderer, M., Büchler, M., Johns, M., Brucker, A. D., Breu, R., \& Pretschner, A. (2016). Chapter one-security testing: A survey. Advances in Computers, 101, 1-51.

[3] Hamisi, N.Y., Mvungi, N.H., Mfinanga, D.A. and Mwinyiwiwa, B.M.M., "Intrusion detection by penetration test in an organization network", ICAST 2009.

[4] Kaur, M. S., \& Singh, M. S. (2016). Penetration testing management. International Journal of Advanced Research in Computer and Communication Engineering, 5(3), 171-177.

[5] Klı'ma, T. (2016). PETA: Methodology of information systems security penetration testing. Acta Informatica Pragensia, 5(2), 98-117.

[6] Mattadi, E., \& Kumar, K. V. (2015). Evaluation of penetration testing and vulnerability assessments. International Journal of Electronics Communication and Computer Engineering, 6(5), 144-148.

[7] Pritchett Willie L, S. D. (2013). Kali Linux Cookbook. Birmingham,UK: Packt Publishing Ltd.

[8] Endraca, A, King, B., Nodalo, G., Maria, M. S., \& Sabas, I.(2013). Web Application Firewall (WAF). International Journal of eEduciation, e-Business, e-Management and e-Learning

[9] Pritchett Willie L, S. D. (2013). Kali Linux Cookbook. Birmingham,UK: Packt Publishing Ltd.

[10] Muniz Jospeh, L. A. (2013). Web Penetration Testing with Linux. Birmingham, UK: Packt Publishing Ltd.

[11] A.K. Kyaw, F. Sioquim, and J. Joseph, "Dictionary attack on Wordpress: Security and forensic analysis," in 2015 2nd International Conference on Information Security and Cyber Forensics, InfoSec 2015, 2016, p. 158-164.

[12] S.P. Ganesh and G. Anandhi, "Database Security: A Study on Threats And Attacks", International Journal on Recent and Innovation Trends in Computing and Communication, vol. 4(6), pp. 512-513, 2015. 